\title{
Optimal spectrum access and power control of secondary users in cognitive radio networks
}

\author{
Yang Yang ${ }^{1}$ (D), Linglong Dai ${ }^{1}$, Jianjun $\mathrm{Li}^{2}$, Shahid Mumtaz ${ }^{3}$ and Jonathan Rodriguez ${ }^{3}$
}

\begin{abstract}
In future $5 \mathrm{G}$ communication system, radio resources can be effectively reused by cognitive radio networks (CRNs), where a lot of secondary users (SUs) are able to access the spectrum of primary users (PUs). In this paper, we analyze the optimal spectrum access and power control of SUs on multiple bands with the target of maximizing the average sum rate (ASR) of SUs. Specifically, based on the stochastic geometry, the random distributions of PUs and SUs are modeled by Poisson point processes (PPPs), based on which we derive out the closed-form outage probabilities and obtain the ASR of SUs. Then, we formulate the maximization problem of ASR on multiple bands under the constraints of outage probabilities. With the help of convex optimization, the optimal density of SUs is obtained in closed-form when the power of SUs is fixed. The convexity of ASR is also verified, and we evaluate the optimal power of SUs when the density of SUs is fixed. Based on these two obtained results, a spectrum access and power control algorithm is further proposed to maximize the ASR of SUs on multiple bands. Simulation results demonstrate that the proposed algorithm can achieve a higher maximum ASR of SUs over the average power allocation algorithm, and the density and power boundary of SUs are constrained by PUs as well as the interference in the networks.
\end{abstract}

Keywords: Cognitive radio networks, Spectrum access, Power control, Poisson point process, Convex optimization

\section{Introduction}

The rapid growth of mobile traffic and the explosion of mobile users makes spectrum shortage more and more serious [1]. Cognitive radio networks (CRNs) are one of the most promising solution to improve spectrum efficiency, which can effectively alleviate the traffic demands by reusing the spectrum of primary users (PUs). In CRNs, secondary users (SUs) are able to access the spectrum of PUs and transmit signals without causing serious interference to PUs [2]. CRNs also brings a lot of benefits such as improving data rate, reducing power consumption, and enhancing spectral efficiency, which make it become an important part for future 5G wireless communications [3].

In CRNs, the random distribution of a large number of SUs significantly aggravate the interference caused by

\footnotetext{
*Correspondence: daill@tsinghua.edu.cn

${ }^{1}$ Tsinghua National Laboratory for Information Science and Technology

(TNList), Department of Electronic Engineering, Tsinghua University, 100084 Beijing, China

Full list of author information is available at the end of the article
}

spectrum reuse. It is very difficult to model the dedicated interference for every SU in random geographical distribution, especially when the number of SUs is very large. Many research works have been proposed to model and analyze the CRNs with Poisson point process (PPP), which different network performances such as signal to interference plus noise ratio (SINR) distribution [4], coverage probability [5], and average spectral efficiency [6]. However, those performance analyses highly depend on the CRNs which PUs and SUs have already accessed the networks. Moreover, if the number of SUs is large, the optimization of spectrum access for performance enhancement in CRNs is challenging [7].

On the other hand, SUs accessing the spectrum of PUs makes the power control in CRNs difficult to operate, especially when multiple frequency spectrum bands are considered. This is due to the factor that different number of users may access different bands, and each user uses different powers to transmit signal on different bands. Moreover, each terminal in CRNs has its own 
power limitation, and the reliability of the PUs' transmission should also be guaranteed. Therefore, power control is necessary to improve spectral efficiency [8] and network throughput [9] and reduce the interference in CRNs [10]. However, combining the spectrum access and power control of SUs to enhance the network performance, particularly on multiple bands with power limitation and random distribution in CRNs, is still needing further investigation.

\subsection{Related works}

Spectrum access is very important for SUs in CRNs, since the communication of PUs in CRNs must be protected at first [11]. Some early research works focused on the spectrum access in the uplink [12] and downlink [13] of PUs, where both the control and the scheduling were managed by the base stations (BSs) in CRNs. Based on these works, a cooperative spectrum sense of other SUs was introduced to reduce the false detection caused by shadowing fading [14]. Furthermore, it was proposed that both PUs and SUs can operate spectrum access in CRNs to improve the transmission rate, where the network connectivity and flexibility can be also enhanced [15].

While there have been considerable works on spectrum access scheduled by some central operator such as BSs in CRNs, very little attention has been paid to the spectrum access under random conditions. The authors in [16] adopted the random spectrum access of SUs in CRNs, where the network latency can be reduced by some extra MAC layer information. Besides, a hybrid spectrum access method was investigated in [17], where both opportunistic spectrum access and exclusive spectrum access were used to optimize the network performance. In addition, some spectrum access schemes were also extended to multiple bands [18], relay scenarios [19], and so on. However, most of the existing works considered the spectrum access in only one or several cells, while the spectrum access of SUs in a large area, especially for the condition with large random geographical user distribution, which still requires further investigation.

\subsection{Contributions}

This study aims to optimize the spectrum access and power control of SUs for maximizing the average sum rate (ASR) in CRNs. Different from most of the existing works in the literature, we not only model the random distribution of the PUs and SUs by PPPs but also consider that the SUs can access the frequency spectrum of PUs on multiple bands. We evaluate the optimal density and power of SUs with outage probabilities and power and density constraints. Based on the analysis, we also propose an algorithm which considers both the spectrum access and the power control of SUs. More specifically, the contributions of this work are summarized as follows:
- With the assumption of random distribution of both PUs and SUs in CRNs, we model the system as PPPs and use Laplace transformation to derive the outage probabilities of both PUs and SUs on one single band. These results show the requirements to the transmission of PUs and SUs, which are also the basic constraints for optimizing the spectrum access of SUs. Note that our derived results can be further optimized on multiple bands, which is more generalized and can include many previously published works $[4,5,15,20,21]$ as special cases only on one single bands.

- The power control is optimized for maximizing ASR of SUs in CRNs. Our analysis not only considers the constraints of outage probabilities, power, and density on multiple bands, but also combines the optimal spectrum access of SUs. We further prove the convexity of ASR in the power definition domain of a secondary network. Then, we get the optimal density of SUs and optimal power of SUs on each bands. Based on these results, a spectrum access and power control algorithm is proposed, which considers the joint interaction effect between density and power of SUs. Simulation results demonstrate that the proposed algorithm can achieve a higher maximum ASR of SUs over the average power allocation algorithm. In addition, the density and power boundaries are constrained by PUs as well as the interference in the networks, and the ASR of SUs can be impacted by different powers of PUs. This new method is different from the previous works only consider power control on multiple bands [16, 17, 22].

\subsection{Outline}

The rest of the paper is organized as follows. Section 2 describes the scenario and network model. Section 3 presents the outage probabilities and the definition of ASR of SUs on multiple bands. In Section 4, we derived the optimal density and power of SUs which access the spectrum of PUs. Moreover, a spectrum access and power control algorithm is proposed to get the maximum ASR of SUs. Simulation results are shown in Section 5. Last, the conclusions are summarized in Section 6.

\section{Scenario description and network model}

In this section, we first describe the scenario of the cognitive radio networks, and then we model the networks with PPPs. Last, the propagation model is explained in detail.

\subsection{Scenario description}

As shown in Fig. 1, the CRNs contain the primary network $S_{1}$ and the secondary network $S_{0}$. The primary networks are deployed on $N$ multiple independent bands, the bandwidths are denoted as $W_{i}, i=1,2, \ldots N$. SUs 


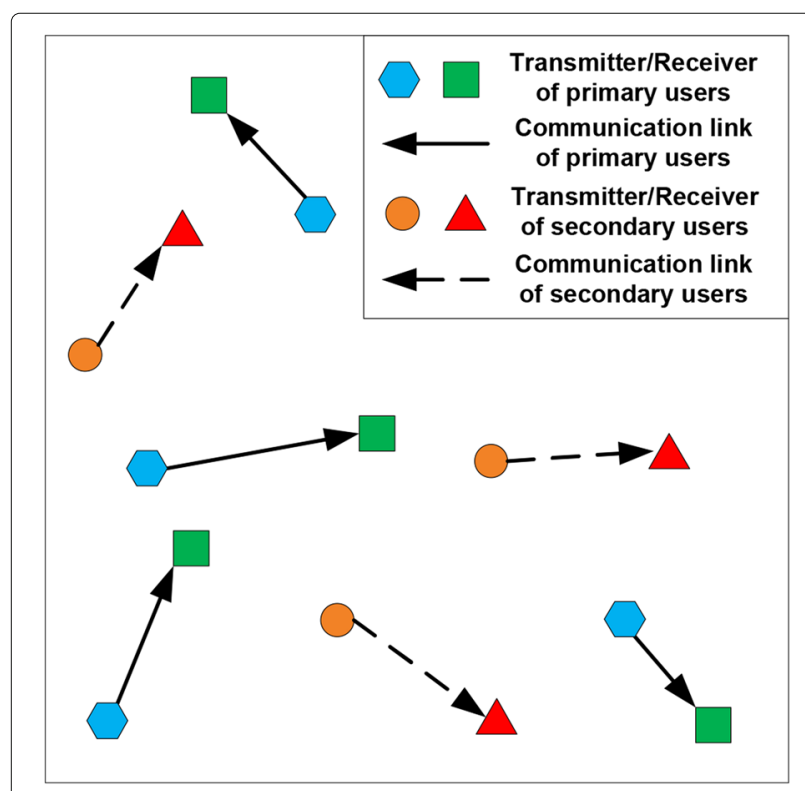

Fig. 1 Scenario of cognitive radio networks

can access the frequency spectrum of PUs. The SUs are under power control because the communication of the PUs cannot be seriously interfered. On each band, the frequency spectrum of the primary network is divided into several frequency-flat sub-channels by utilizing an orthogonal frequency-division multiplexing technology. The full set of the sub-channels can be reused by SUs as an underlay sharing with the primary network.

\subsection{Network models}

Based on stochastic geometry theory, following assumptions are made:

Assumption 1 The transmitters of secondary network form a PPP on the two dimensional plane $\Re$, which is denoted as $\Pi_{0}$ with the density $\lambda_{0, i}$ on band $i, i=$ $1,2, \ldots, N$. The transmission powers of the SUs are denoted as $P_{0, i},(i=1,2, \ldots, N)$ on each band.

Assumption 2 The transmitters of PUs form a series of stationary PPPs on each band, which are denoted as $\Pi_{1}^{i}$ with the density $\lambda_{1, i},(i=1,2, \ldots, N)$ on $\Re$. The transmission powers of the PUs are denoted as $P_{1, i},(i=1,2, \ldots, N)$ on each band.

Assumption 3 According to Palm theory [23], a typical receiver of $S_{j}, j \in\{0,1\}$ is located at the origin, which does not influence the statistics of the PPP.

\subsection{Propagation models}

We consider both path loss and Rayleigh fading as the propagation models with the following form:

$$
P_{r x}=\delta P_{t x}|D|^{-\alpha},
$$

where $P_{t x}$ and $P_{r x}$ represent the transmitter and receiver power, respectively, $\alpha$ is the path loss exponent, $|D|$ is the distance between the transmitter and the receiver. $\delta$ stands for Rayleigh fading coefficient, which has an independent exponential distribution with unit mean for every communication link in the CRNs.

When SUs access the spectrum of PUs, the receiver suffers from the interference generated by transmitters in both primary and secondary networks. The distribution of the interfering users in a single network $S_{j}, j \in\{0,1\}$ on band $i$ can be modeled by mark PPP, which is denoted as $\Pi_{j}=\left\{\left(X_{j k}, \delta_{j k}\right)\right\}$, where $\delta_{j k}$ and $X_{j k}$ are Rayleigh fading and the distance from the origin to the node $k$ of network $S_{j}$.

\section{Average sum rate of secondary network on multi-bands}

\subsection{The outage probability on one single band}

The interference received at a typical receiver is generated by both primary and secondary networks occupying the specific band, the SINR of network $S_{n}$, ( $n$ is 0 or 1$)$ on the $i$ th band at the receiver is

$$
\operatorname{SINR}_{n, i}=\frac{P_{n, i} \delta_{n 0, i} R_{n 0, i}^{-\alpha}}{\sum_{j \in\{0,1\}} \sum_{\left(X_{j k}, \delta_{j k}\right) \in \Pi_{j}} P_{j, i} \delta_{j k}\left|X_{j k}\right|^{-\alpha}+N_{0}},
$$

where $\delta_{n 0, i}$ and $R_{n 0, i}$ are the Rayleigh fading and the distance from the desired transmitter to the typical receiver of network $S_{n}$ on the $i$ th band, respectively. $N_{0}$ is the thermal noise. Because the spectrum access of SUs is our main consideration, which means that the CRNs are interference limited, the thermal noise is negligible. Then, SINR is replaced by SIR (signal to interference ratio) as follows:

$$
\operatorname{SIR}_{n, i}=\frac{\delta_{n 0, i} R_{n 0, i}^{-\alpha}}{I_{n, i, 0}+I_{n, i, 1}}
$$

where $I_{n, i, 0}=\sum_{\left(X_{0 k}, \delta_{0 k}\right) \in \Pi_{0}}\left(\frac{P_{0, i}}{P_{n, i}}\right) \delta_{0 k}\left|X_{0 k}\right|^{-\alpha}, I_{n, i, 1}=$ $\sum_{\left(X_{1 k}, \delta_{1 k}\right) \in \Pi_{1}}\left(\frac{P_{1, i}}{P_{n, i}}\right) \delta_{1 k}\left|X_{1 k}\right|^{-\alpha}$. Set $T_{n, i}$ as the threshold of SIR on the $i$ th band, the following lemma shows the outage probability of a typical receiver:

Lemma 1 The outage probability of a typical receiver of $S_{n}$, ( $n$ is 0 or 1$)$ on the ith band $(i=1,2, \ldots, N)$ satisfies

$$
\left.\operatorname{Pr}\left(\operatorname{SIR}_{n, i} \leq T_{n, i}\right)=1-e^{\left\{-\varsigma_{n, i} \sum_{j \in\{0,1\}} \lambda_{j, i}\left(\frac{P_{j, i}}{P_{n, i}}\right)^{\frac{2}{\alpha}}\right.}\right\},
$$

where $\operatorname{Pr}(\bullet)$ represent the probability, $\varsigma_{n, i}=[\pi \Gamma$ $\left.\left(1+\frac{2}{\alpha}\right) \Gamma\left(1-\frac{2}{\alpha}\right)\right] T_{n, i}^{\frac{2}{\alpha}} R_{n 0, i}^{2}$ 
Proof From Eq. (3), the outage probability satisfies:

$$
\begin{aligned}
\operatorname{Pr} & \left(S I R_{n, i} \leq T_{n, i}\right) \\
& =1-\operatorname{Pr}\left(S I R_{n, i} \geq T_{n, i}\right) \\
& =1-\operatorname{Pr}\left(\delta_{n 0, i} \geq T_{n, i} R_{n 0, i}^{\alpha}\left(I_{n, i, 0}+I_{n, i, 1}\right)\right) \\
& \left.=1-\int_{0}^{\infty} e^{-S T_{n, i} R_{n 0, i}^{\alpha}} d \operatorname{Pr}\left(I_{n, i, 0}+I_{n, i, 1} \leq s\right)\right] \\
& =1-\psi_{I_{n, i, 0}}\left(T_{n, i} R_{n 0, i}^{\alpha}\right) \psi_{I_{n, i, 1}}\left(T_{n, i} R_{n 0, i}^{\alpha}\right)
\end{aligned}
$$

where $\psi_{I_{n, i, 0}}(\bullet)$ and $\psi_{I_{n, i, 1}}(\bullet)$ are Laplace transformation of $I_{n, i, 0}$ and $I_{n, i, 1}$, respectively. Because the analysis is based on the two dimensional planes, $\delta_{n 0, i}$ satisfies an independent exponential distribution and we have [24]

$$
\left.\psi_{I_{n, i, 0}}(s)=e^{\left\{-\lambda_{0, i} \pi\left(\frac{s P_{0, i}}{P_{n, i}}\right)^{\frac{2}{\alpha}} \Gamma\left(1+\frac{2}{\alpha}\right) \Gamma\left(1-\frac{2}{\alpha}\right)\right.}\right\} .
$$

Here, $\Gamma(\bullet)$ is the gamma function with the form $\Gamma(z)=$ $\int_{0}^{\infty} e^{-t} t^{z-1} d t$. Similarly,

$$
\psi_{I_{n, i, 1}}(s)=e^{\left\{-\lambda_{1, i} \pi\left(\frac{s P_{1, i}}{P_{n, i}}\right)^{\frac{2}{\alpha}} \Gamma\left(1+\frac{2}{\alpha}\right) \Gamma\left(1-\frac{2}{\alpha}\right)\right\}} .
$$

Substitute (6) and (7) back into (5), the result is

$$
\begin{aligned}
\operatorname{Pr} & \left(S_{R} R_{n, i} \leq T_{n, i}\right) \\
& =1-e^{\left\{-\pi \Gamma\left(1+\frac{2}{\alpha}\right) \Gamma\left(1-\frac{2}{\alpha}\right) T_{n, i}^{\frac{2}{\alpha}} R_{n 0, i}^{2} \sum_{j \in\{0,1\}} \lambda_{j, i}\left(\frac{P_{j, i}}{P_{n, i}}\right)^{\frac{2}{\alpha}}\right\} .}
\end{aligned}
$$

Denote $\varsigma_{n, i}=\pi \Gamma\left(1+\frac{2}{\alpha}\right) \Gamma\left(1-\frac{2}{\alpha}\right) T_{n, i}^{\frac{2}{\alpha}} R_{n 0, i}^{2}$, Eq. (4) is obtained.

Based on Lemma 1, the successful transmission probability of a typical receiver of $S_{n}$, ( $n$ is 0 or 1 ) on the $i$ th band $(i=1,2, \ldots, N)$ is expressed as

$$
\begin{aligned}
& \operatorname{Pr}_{n, i}^{\text {suc }}\left(\lambda_{n, i}, \lambda_{j, i}\right) \\
& \quad=1-\operatorname{Pr}\left(\operatorname{SIR}_{n, i} \leq T_{n, i}\right) \\
& \quad=\operatorname{Pr}\left(\operatorname{SIR}_{n, i} \geq T_{n, i}\right),
\end{aligned}
$$

where $\lambda_{n, i}$ is the node density of $S_{n}$ on the $i$ th band.

\subsection{Average sum rate of the secondary network on multiple bands}

In the CRNs, the ASR of the secondary network on multiple bands is defined as [25]:

$$
f\left(\lambda_{0, i}, P_{0, i}\right) \quad=\sum_{i=1}^{N} \omega_{i} \lambda_{0, i} e^{-\zeta_{0, i}\left[\lambda_{0, i}+\left(\frac{P_{1, i}}{P_{0, i}}\right)^{\frac{2}{\alpha}} \lambda_{1, i}\right]},
$$

where $\omega_{i}=\frac{W_{i}}{\sum_{m=1}^{N} W_{m}}, W_{i}$ is the bandwidth of the $i$ th band which is reused by SUs, $P_{0, i}$ is the power of SUs, and $\lambda_{0, i}$ is the density of SUs on the $i$ th band.
The communication of PUs must be protected when SUs access the spectrum. Then, we get the following constraints:

$$
\begin{aligned}
& 1-e^{-\zeta_{0, i}\left[\lambda_{0, i}+\left(\frac{P_{1, i}}{P_{0, i}}\right)^{\frac{2}{\alpha}} \lambda_{1, i}\right]} \leq \theta_{0}, \\
& 1-e^{-\zeta_{1, i}\left[\lambda_{1, i}+\left(\frac{P_{0, i}}{P_{1, i}}\right)^{\frac{2}{\alpha}} \lambda_{0, i}\right]} \leq \theta_{1}, \\
& 0 \leq \lambda_{0, i} \leq \lambda_{\max , i},(i=1,2, \ldots, N), \\
& 0 \leq P_{0, i} \leq P_{\max , i},(i=1,2, \ldots, N),
\end{aligned}
$$

where $\theta_{0}$ and $\theta_{1}$ are the outage probability thresholds of SUs and PUs, respectively. $\lambda_{\max , i}$ and $P_{\max , i}$ are the maximum density and power of SUs on each band, respectively.

\section{Maximizing the ASR of the secondary network on multiple bands}

In this section, the ASR of the secondary network on multiple bands is maximized under the density and power constraints. Then, we get the optimal density and power of SUs in closed-form. Last, a spectrum access and power control algorithm is proposed to get the maximum ASR of the secondary network.

\subsection{Maximizing ASR of the secondary network on multi-bands with density constraints}

First, we fix the power of SUs. Then, from (11) and (12), we have:

$$
\begin{aligned}
& \lambda_{0, i} \leq \frac{-1}{\zeta_{0, i}} \ln \left(1-\theta_{0}\right)-\left(\frac{P_{1, i}}{P_{0, i}}\right)^{\frac{2}{\alpha}} \lambda_{1, i}, \\
& \lambda_{0, i} \leq\left(\frac{P_{1, i}}{P_{0, i}}\right)^{\frac{2}{\alpha}}\left(\frac{-1}{\varsigma_{1, i}} \ln \left(1-\theta_{1}\right)-\lambda_{1, i}\right) .
\end{aligned}
$$

Make $\lambda_{0, i, \text { sup }_{1}}=\frac{-1}{\varsigma_{0, i}} \ln \left(1-\theta_{0}\right)-\left(\frac{P_{1, i}}{P_{0, i}}\right)^{\frac{2}{\alpha}} \lambda_{1, i}$ and $\lambda_{0, i, \sup _{2}}=$ $\left(\frac{P_{1, i}}{P_{0, i}}\right)^{\frac{2}{\alpha}}\left(\frac{-1}{\zeta_{1, i}} \ln \left(1-\theta_{1}\right)-\lambda_{1, i}\right)$, from constraint (13), the upper density limit of SUs on a single band is $\lambda_{0, i \text {,sup }}=$ $\min \left\{\lambda_{0, i, \text { sup }_{1}}, \lambda_{0, i, \text { sup }_{2}}, \lambda_{\max , i}\right\},(i=1,2, \ldots, N)$.

Denoting the density of SUs as $\lambda_{0}$, we get $\sum_{i=1}^{N} \lambda_{0, i}=\lambda_{0}$ and $\lambda_{0} \leq \sum_{i=1}^{N} \lambda_{0, i, \text { sup }}$. While $\lambda_{0}>\sum_{i=1}^{N} \lambda_{0, i \text {,sup }}$, the network should control the spectrum access of SUs to satisfy $0 \leq$ $\lambda_{0, i} \leq \lambda_{0, i, \text { sup }},(i=1,2, \ldots, N)$. Then, we get the following two conditions: 
(1) When $\lambda_{0}>\sum_{i=1}^{N} \lambda_{0, i, \text { sup }}$, we have

$$
\begin{aligned}
& \max f\left(\lambda_{0, i}, P_{0, i}\right)=\sum_{i=1}^{N} \omega_{i} \lambda_{0, i} e^{-\zeta_{0, i}\left[\lambda_{0, i}+\left(\frac{P_{1, i}}{P_{0, i}}\right)^{\frac{2}{\alpha}} \lambda_{1, i}\right]} \\
& \text { s.t. } \quad 0 \leq \lambda_{0, i} \leq \lambda_{0, i, \text { sup }},(i=1,2, \ldots, N) .
\end{aligned}
$$

Taking the partial derivation of $f\left(\lambda_{0, i}, P_{0, i}\right)$ with respect to $\lambda_{0, i}$,

$$
\frac{\partial f\left(\lambda_{0, i}, P_{0, i}\right)}{\partial \lambda_{0, i}}=\left(1-\varsigma_{0, i} \lambda_{0, i}\right) \omega_{i} e^{-\zeta_{0, i}\left[\lambda_{0, i}+\left(\frac{P_{1, i}}{P_{0, i}}\right)^{\frac{2}{\alpha}} \lambda_{1, i}\right]} .
$$

Making $\frac{\partial f\left(\lambda_{0, i}, P_{0, i}\right)}{\partial \lambda_{0, i}}=0, \lambda_{0, i}=\frac{1}{\zeta_{0, i}}$ is obtained, so the optimal density of SUs on the $i$ th band $\lambda_{0, i, o p t 1}^{*}$ is

$$
\lambda_{0, i, o p t 1}^{*}=\left\{\begin{array}{cl}
\lambda_{0, i \text {,sup }} & \lambda_{0, i, \text { sup }}<\frac{1}{\rho_{0, i}} \\
\frac{1}{\varsigma_{0, i}} & \lambda_{0, i, \text { sup }} \geq \frac{1}{\varsigma_{0, i}}
\end{array}(i=1,2, \ldots, N) .\right.
$$

(2) When $\lambda_{0} \leq \sum_{i=1}^{N} \lambda_{0, i \text {,sup }}$, we have

$$
\begin{aligned}
\max f\left(\lambda_{0, i}, P_{0, i}\right)= & \sum_{i=1}^{N} \omega_{i} \lambda_{0, i} e^{-\zeta_{0, i}\left[\lambda_{0, i}+\left(\frac{P_{1, i}}{P_{0, i}}\right)^{\frac{2}{\alpha}} \lambda_{1, i}\right]} \\
\text { s.t. } \quad 0 \leq \lambda_{0, i} \leq & \lambda_{0, i, \text { sup }},(i=1,2, \ldots, N) \\
& \sum_{i=1}^{N} \lambda_{0, i}=\lambda_{0}
\end{aligned} .
$$

Then, we get the optimal density of SUs in the following theorem:

Theorem 1 When the power of SUs on the ith band $(i=1,2, \ldots, N)$ is fixed, the optimal density of SUs on the ith band $\lambda_{0, i, o p t 2}^{*}$ is

$$
\lambda_{0, i, o p t 2}^{*}=\left\{\begin{array}{cc}
\lambda_{0, i \text { sup }}, & 0 \leq \psi<\psi_{u} \\
\frac{(1-\sqrt{\rho})}{50_{0}}, & \psi_{u} \leq \rho<1 \\
0, & 1 \leq \rho
\end{array},\right.
$$

where $\rho=\frac{v}{A_{i}}, A_{i}=\omega_{i} e^{-\zeta_{0, i}\left(\frac{P_{1, i}}{P_{0, i}}\right)^{\frac{2}{\alpha}}} \lambda_{1, i},(i=1,2, \ldots, N)$.

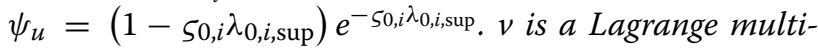
plier coefficient which satisfies $\sum_{i=0}^{N} \lambda_{0, i, o p t 2}^{*}=\lambda_{0}$.

Proof See Appendix 1.

\subsection{Maximizing the ASR of the secondary network on multi-bands with power constraints}

In the following, we will maximize the ASR of the secondary network with the power constraint of SUs. Similarly, we fixed the density of SUs. According to (11) and (12), we have

$$
\begin{aligned}
& P_{0, i} \geq P_{1, i}\left[\frac{-\ln \left(1-\theta_{0}\right)}{\lambda_{1, i} S_{0, i}}-\frac{\lambda_{0, i}}{\lambda_{1, i}}\right]^{\frac{-\alpha}{2}}, \\
& P_{0, i} \leq P_{1, i}\left[\frac{-\ln \left(1-\theta_{1}\right)}{\lambda_{0, i} S_{1, i}}-\frac{\lambda_{1, i}}{\lambda_{0, i}}\right]^{\frac{\alpha}{2}} .
\end{aligned}
$$

Denote $P_{0, i, \text { inf }_{1}}=P_{1, i}\left[\frac{-\ln \left(1-\theta_{0}\right)}{\lambda_{1, i} S_{0, i}}-\frac{\lambda_{0, i}}{\lambda_{1, i}}\right]^{\frac{-\alpha}{2}}$ and $P_{0, i, \text { sup }_{1}}=$ $P_{1, i}\left[\frac{-\ln \left(1-\theta_{1}\right)}{\lambda_{0, i} S_{1, i}}-\frac{\lambda_{1, i}}{\lambda_{0, i}}\right]^{\frac{\alpha}{2}}$, from constraint (14), the lower and upper power limit of SUs in a single band are $P_{0, i, \text { inf }}=$ $\max \left\{0, P_{0, i, \text {,inf }_{1}}\right\}$ and $P_{0, i \text {,sup }}=\min \left\{P_{\max , i}, P_{0, i, \text { sup }_{1}}\right\}, i=$ $1,2, \ldots, N$, respectively.

Define $P_{0}$ as the power for one SUs on all the bands, so we have $P_{0} \leq \sum_{i=1}^{N} P_{0, i \text { sup }}$ otherwise, we can control the spectrum access of SUs on each band to make $P_{0, i, \text { inf }} \leq P_{0, i} \leq P_{0, i, \text { sup }},(i=1,2, \ldots, N)$ established when $P_{0}>\sum_{i=1}^{N} P_{0, i, \text { sup }}$. Similarly, we get following two aspects:

(1) When the power constraint $P_{0}>\sum_{i=1}^{N} P_{0, i, \text { sup }}$, we have

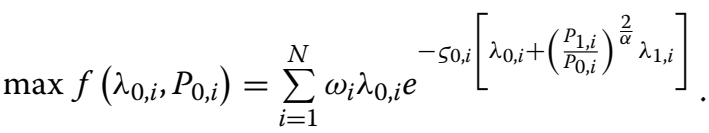

$$
\begin{aligned}
& \text { s.t. } \quad P_{0, i, \text { inf }} \leq P_{0, i} \leq P_{0, i, \text { sup }},(i=1,2, \ldots, N)
\end{aligned}
$$

When $P_{0, i}=P_{0, i, \text { sup }}, f\left(\lambda_{0, i}, P_{0, i}\right)$ can reach the maximum value in the definition domain of $P_{0, i}$, we get the optimal power of SUs on the $i$ th band as:

$$
P_{0, i, o p t 1}^{*}=P_{0, i, \text { sup }},(i=1,2, \ldots, N) .
$$

According to the above analysis, we can the maximize value of ASR only when $P_{0, i, \text { inf }} \leq P_{0, i, \text { sup }},(i=1,2, \ldots, N)$. But when $P_{0, i, \text { inf }}>P_{0, i \text {,sup }}$ on the $i$ th band, the following inequality holds:

$$
\left[\frac{-\ln \left(1-\theta_{0}\right)}{\lambda_{1, i} \varsigma_{0, i}}-\frac{\lambda_{0, i}}{\lambda_{1, i}}\right]^{\frac{-\alpha}{2}}>\left[\frac{-\ln \left(1-\theta_{1}\right)}{\lambda_{0, i} \varsigma_{1, i}}-\frac{\lambda_{1, i}}{\lambda_{0, i}}\right]^{\frac{\alpha}{2}} .
$$

Let $\xi_{0, i}=\frac{-\ln \left(1-\theta_{0}\right)}{\varsigma_{0, i}}$ and $\xi_{1, i}=\frac{-\ln \left(1-\theta_{1}\right)}{\varsigma_{1, i}}$, then reshape (26), we have

$$
\left(\frac{\xi_{0, i}}{\lambda_{1, i}}-\frac{\lambda_{0, i}}{\lambda_{1, i}}\right)\left(\frac{\xi_{1, i}}{\lambda_{0, i}}-\frac{\lambda_{1, i}}{\lambda_{0, i}}\right)<1 .
$$


Remark 1 When more SUs access the spectrum, the interference is becoming more and more serious. Once the density of SUs on the ith band is large enough to make inequality (27) established, the communication of the PUs cannot be ensured if SUs transmit signal on that band. Then power control must be operated, so $P_{0, i}$ should be zero under this condition.

(2) When the power constraints of SUs $P_{0}<\sum_{i=1}^{N} P_{0, i, \text { sup }}$, we have

$$
\begin{array}{cc}
\max f\left(\lambda_{0, i}, P_{0, i}\right)= & \sum_{i=1}^{N} \omega_{i} \lambda_{0, i} e^{-\zeta_{0, i}\left[\lambda_{0, i}+\left(\frac{P_{1, i}}{P_{0, i}}\right)^{\frac{2}{\alpha}} \lambda_{1, i}\right]} \\
\text { s.t. } \quad P_{0, i, \text { inf }} \leq P_{0, i} \leq P_{0, i, \text { sup }},(i=1,2, \ldots, N) & \sum_{i=1}^{N} P_{0, i}=P_{0}
\end{array} .
$$

In cognitive radio networks, the outage probability threshold of PUs $\theta_{1}$ is usually defined as a very small value because the priority of PUs should be provided. Then, denoting $B_{i}=\omega_{i} \lambda_{0, i} e^{-\zeta_{0, i} \lambda_{0, i}, D_{i}}=\varsigma_{0, i} P_{1, i}^{\frac{2}{\alpha}} \lambda_{1, i},(i=$ $1,2, \ldots, N)$, we get the following lemma and theorem:

Lemma 2 When outage probability threshold is $\theta_{1} \in$ $\left(0,1-e^{-\lambda_{1, i} \zeta_{1, i}}\right)$, the negative ASR of secondary network

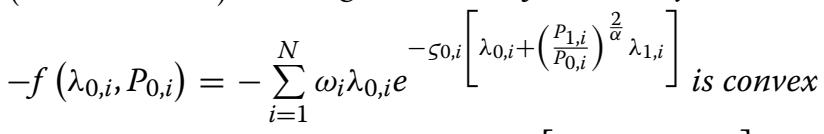
in the power definition domain of SUs $\left[P_{0, i, i n f}, P_{0, i, \text { sup }}\right]$.

Proof See Appendix 2.

Then, following theorem shows the optimal power of SUs in each band:

Theorem 2 When the density of SUs on each band is fixed, the optimal power of SUs in the ith $(i=1,2, \ldots, N)$ band $P_{0, i, o p t 2}^{*}$ is

$$
P_{0, i, \text { opt } 2}^{*}=\left\{\begin{array}{cc}
P_{0, i, \mathrm{sup}}, \quad u \leq h_{0, i, \min } \\
P_{0, i, \text { solution }}^{*}, h_{0, i, \min }<u \leq h_{0, i, \max }, \\
P_{0, i, \text { inf }}, \quad h_{0, i, \max }<u
\end{array}\right.
$$

where $\left[h_{0, i, \min }, h_{0, i, \max }\right]$ is the range of the function $h\left(P_{0, i}\right)=\frac{2 B_{i} D_{i}}{\alpha} e^{-D_{i} P_{0, i}^{\frac{-2}{\alpha}}} P_{0, i}^{-\left(1+\frac{2}{\alpha}\right)}$, and $P_{0, i, s o l u t i o n}^{*}$ is the solution of $u-h\left(P_{0, i}\right)=0$. While $u$ is a Lagrange multiplier coefficient which is determined by the condition $\sum_{i=1}^{N} P_{0, i}=$ $P_{0}$.

Proof See Appendix 3.

\subsection{Spectrum access and power control algorithm for maximizing ASR of the secondary network}

Based on the previous analysis, the ASR of the secondary network is convex when the density or the power of SUs is fixed. Then, we propose a spectrum access and power control algorithm for maximizing ASR of the secondary network. The detail of the algorithm is described in Algorithm 1.

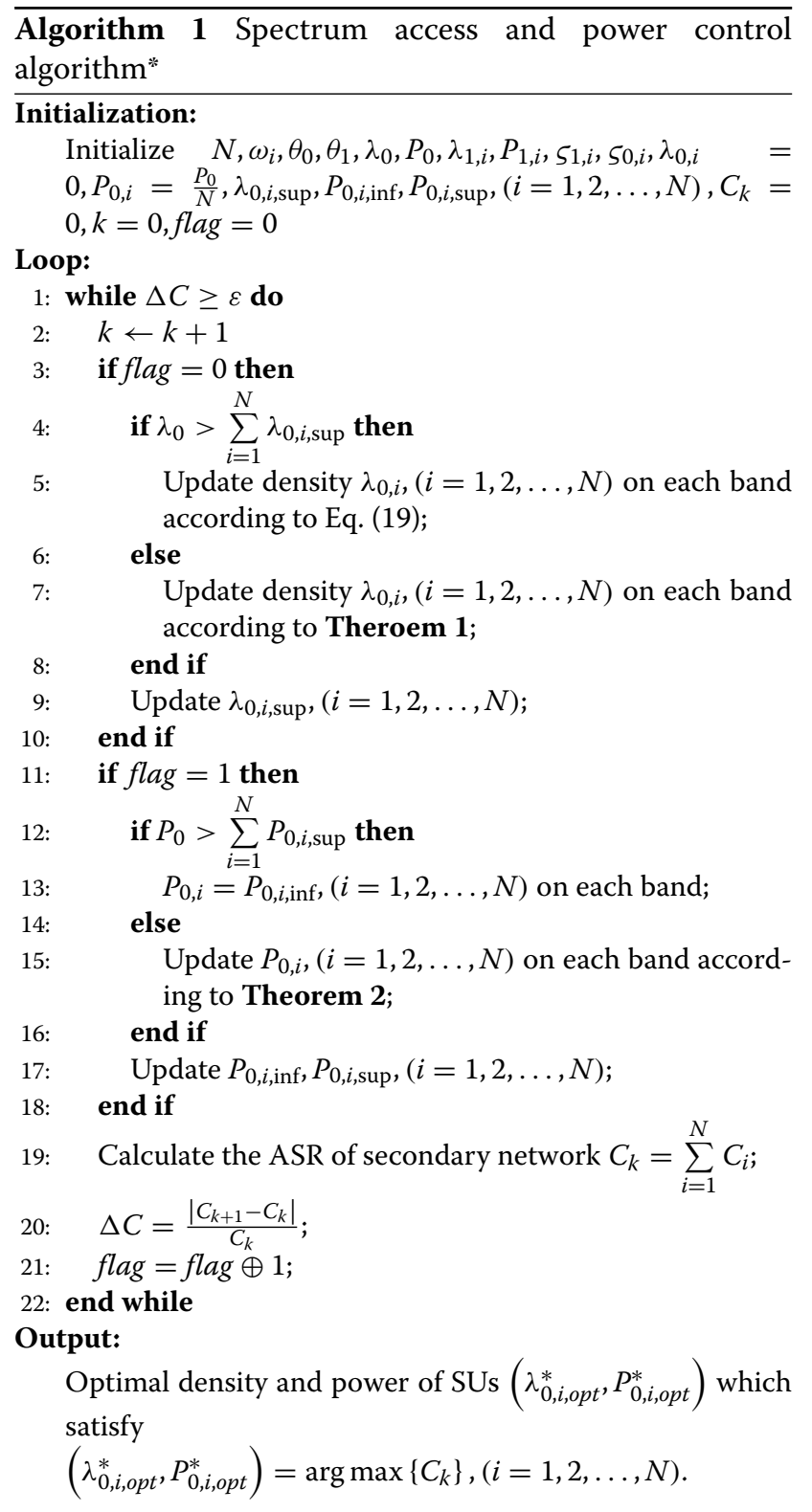

"Note: 1. $C_{k}$ and $C_{i}$ are the ASRs of the secondary network in the $k$ th iteration and $i$ th band, respectively.

2. flag means the flag bit in every iteration which has only the value 0 or 1 .

3. $\varepsilon$ is a pre-defined threshold of $\Delta C$.

4. The operational symbol " $\oplus$ " means logical XOR operation. 
The algorithm aims to use the optimal results in Theorems 1 and 2 to get the optimal spectrum of SUs and the optimal power of SUs, respectively. Several interpretations are shown as follows:

From step 4 to step 8 , the algorithm calculates the optimal density of SUs on each band $(i=1,2, \ldots, N)$, i.e., we can get the optimal number of SUs which access the spectrum of PUs. Step 9 updates the upper density bound of SUs on each band. Then, step 12 to step 16 calculate the optimal power of SUs on each band $(i=1,2, \ldots, N)$, which the calculation is according to the Theorem 2. Step 17 updates the lower and upper bound of SUs on each band.

According to the two if-else-end parts, each time we get the optimal density of SUs, the constraints of the power are changed. Similarly, when we calculate the optimal power of SUs, the value will change the constraints of the density. So, we make an iteration between the optimization between the density and power of SUs, which is controlled by the flag bit in step 21 . We also calculate the ASR gap between each two iterations, which is shown as $\Delta C$ in step 20 . The final optimal density and power of SUs will be reached when $\Delta C<\varepsilon$, where $\varepsilon$ is a pre-defined threshold of $\Delta C$. In the end, we get the optimal density and power of SUs in CRNs.

\section{Simulation results and discussions}

In this section, the outage probability and ASR of secondary network on one single band are analyzed. Then, the density and power boundary which confine the optimization are discussed. Moreover, we present the maximum ASRs of the secondary network on multiple bands. Finally, the maximum ASR on five bands with the proposed algorithm is compared with average power allocation method to make the results more insightful.

\subsection{Simulation analysis of outage probability, density and power boundaries of SUs on the single band}

The basic parameters on one single band are listed in Table 1. We consider a simulation scenario as a $500 \times$ $500 \mathrm{~m}^{2}$ square region. The traffic model is full buffer. Both the number of PUs or SUs on these single bands is set as 25 . The band is assumed with a bandwidth normalized to 1 . The subscript $i$ means the serial number of this band.

Figure 2 illustrates the relationship between the outage probability and the density of SUs on a single band. From Fig. 2, outage probability is rising as the density of SUs is increasing because of higher density of SUs causing more serious interference to the secondary network. Furthermore, Fig. 2 shows the outage probability of SUs under different densities of PUs on a same band. With the increasing density of PUs, the secondary network suffers more interference from the primary network, so the outage probability of SUs is bigger when the density of PUs is higher. Furthermore, the higher the power of SUs is, the lower the outage probability of SUs is. It is known that secondary network can get a better communication quality with a higher power of SUs under the same density of SUs in the CRNs.

In Fig. 3, the relationship between outage probability of SUs and power of SUs on a single band is shown. From Fig. 3, outage probability of SUs is reducing as the power of SUs is increasing because the high power of SUs can bring the improvement of SIR in the secondary network under the same environment. In addition, the figure reveals that the bigger density of SUs makes a higher outage probability of SUs under the same power of SUs. This is due to the interference between SUs that is more serious as the density of SUs is increasing. Moreover, when the power of SUs is increasing, the outage probability of SUs are approaching to stable. This is due to the power of SUs that can only reduce the harmful influence from the primary network to the secondary network, but cannot completely reduce the interference among secondary networks.

Following, Fig. 4 illustrates that the value range of density of SUs, which means how many SUs can access the spectrum of PUs. Two curves with same color are based on Eqs. (15) and (16). Black ellipses mean the density boundaries of SUs, which are the maximum densities of SUs we can choose. Furthermore, SUs can use a lower power when the power of PUs is low because interference from the primary network is small. In addition, when the power of PUs is high, SUs can get bigger density value with a high power of SUs. This is due to the primary network that can bear more interference when the power of PUs is high.

In Fig. 5, power boundaries of SUs on a single band are shown. Black circles point out the intersection of the power upper bound and lower bound. When density of

Table 1 Simulation parameters on one single band

\begin{tabular}{lll}
\hline Parameter & Physical mean & Value \\
\hline$\lambda_{1, i}$ & Density of PUs & $0.0001 \mathrm{user} / \mathrm{m}^{2}$ \\
$\lambda_{0, i}$ & Density of SUs & $0.0001 \mathrm{user} / \mathrm{m}^{2}$ \\
$P_{1, i}$ & Power of PUs & $25 \mathrm{dBm}$ \\
$P_{0, i}$ & Power of SUs & $15 \mathrm{dBm}$ \\
$\alpha$ & Path loss coefficient & 4 \\
$T_{0, i} / T_{1, i}$ & SIR threshold of SUs / PUs & $0 \mathrm{~dB}$ \\
$R_{10, i}$ & Average link distance in the primary & $50 \mathrm{~m}$ \\
$R_{00, i}$ & network & $15 \mathrm{~m}$ \\
$\theta_{0} / \theta_{1}$ & $\begin{array}{l}\text { Average link distance in the } \\
\text { secondary network }\end{array}$ & \\
\hline
\end{tabular}




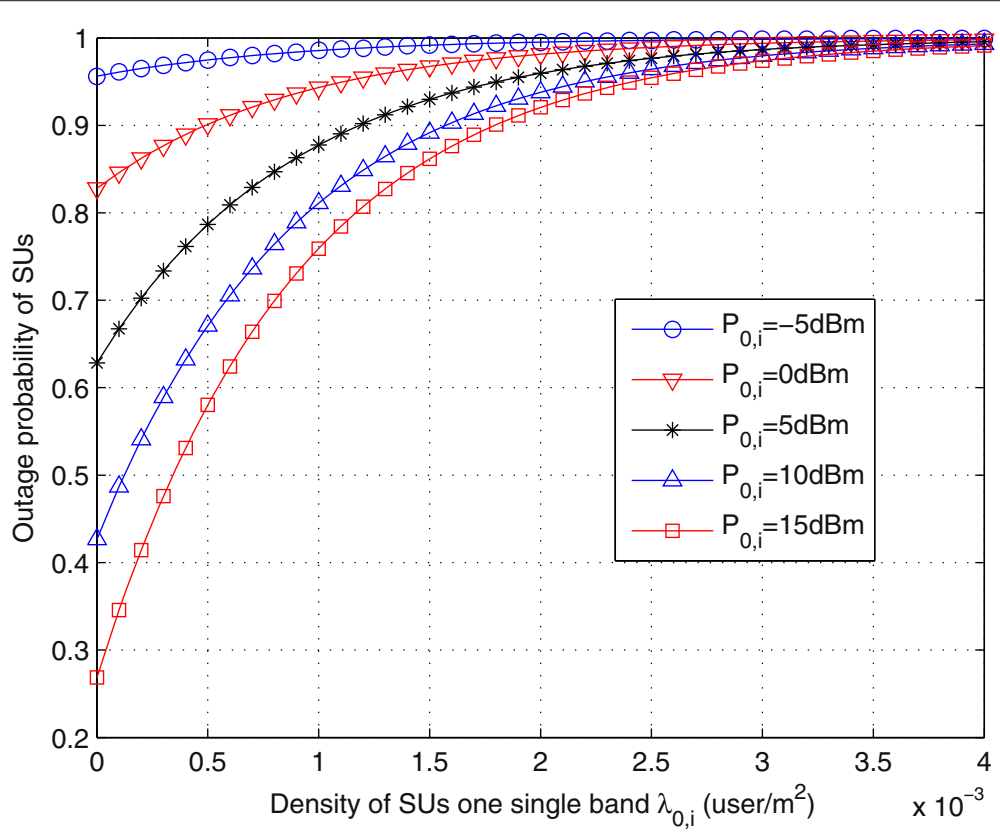

Fig. 2 Outage probability of SUs vs. density of SUs on one single band

SUs exceeds that point, SUs cannot access the spectrum of PUs in order to protect the communication of PUs. From Fig. 5, the boundary curve with bigger power of PUs is higher than the curve with low power. This is due to the primary network that suffers more interference from SUs while the secondary network needs high power to resist interference from the primary network.

\subsection{Simulation analysis of ASR and maximum ASR of the secondary network}

Figure 6 demonstrates the change of ASR of the secondary network with power of SUs on a single band. We can see that the ASR of SUs increases with the power of SUs because the high power of SUs can improve the SIR in the secondary network. More specifically, Fig. 6 also indicates

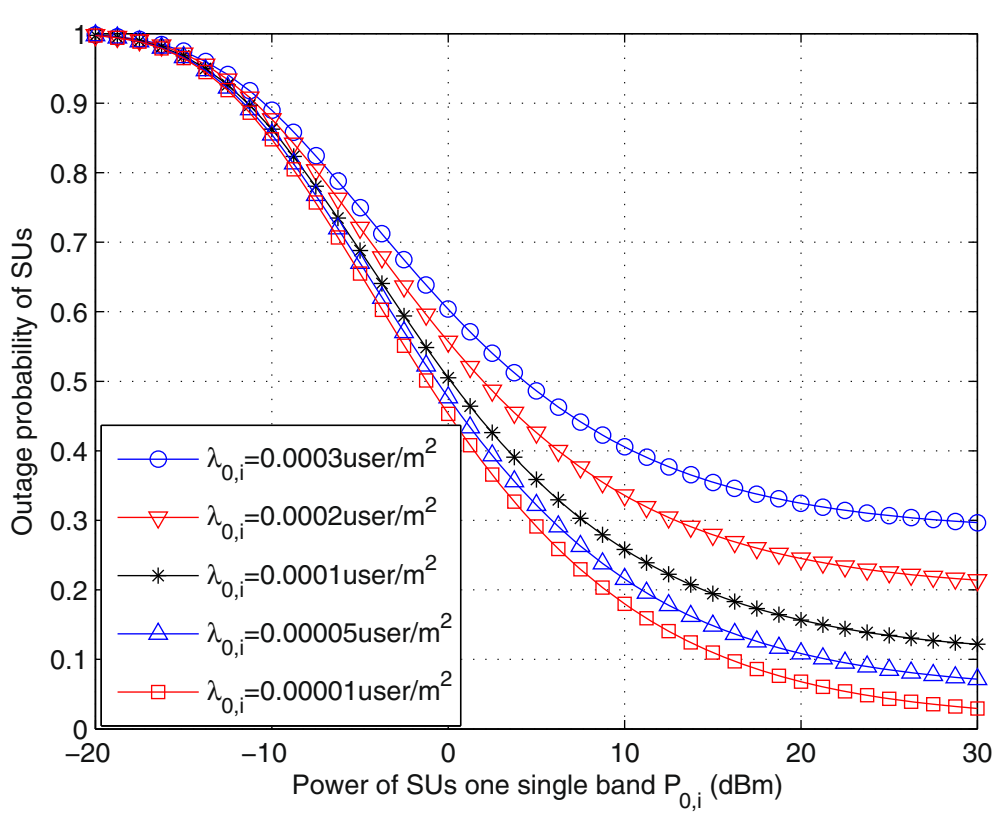

Fig. 3 Outage probability of SUs vs. power of SUs on one single band 


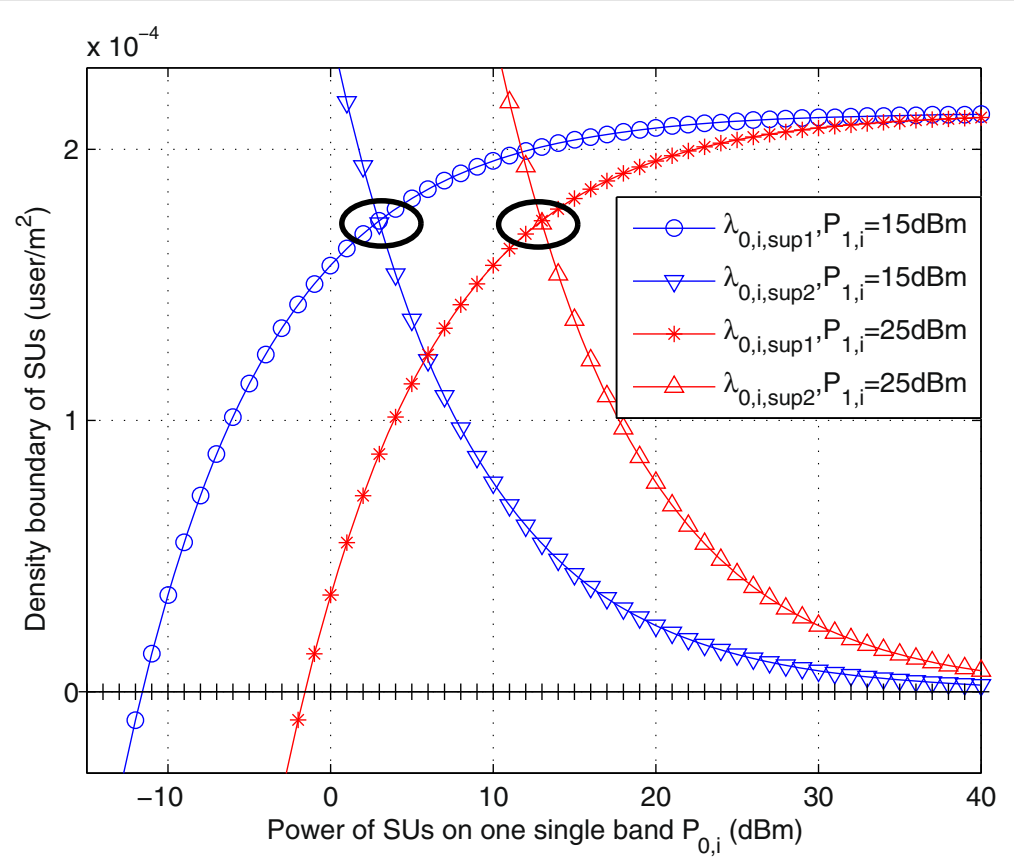

Fig. 4 Density boundary of SUs vs. power of SUs on one single band

that the high density of SUs leads to high ASR of SUs. When the power of SUs is high, the curves tend to stable values because the interference among SUs cannot be completely eliminated by enhancing the power of SUs.

In Fig. 7, the relationship between ASR of the secondary network and density of SUs on a single band is considered. When density of SUs is low, the ASR of the secondary network increases as the density of SUs increases. This is due to more SUs accessing the spectrum of PUs that enhance the performance of the secondary network. When the density of SUs is high and continues to increase, the interference among the SUs becomes large and causes harmful interference to the secondary network, so the ASR of the secondary network begins to reduce.

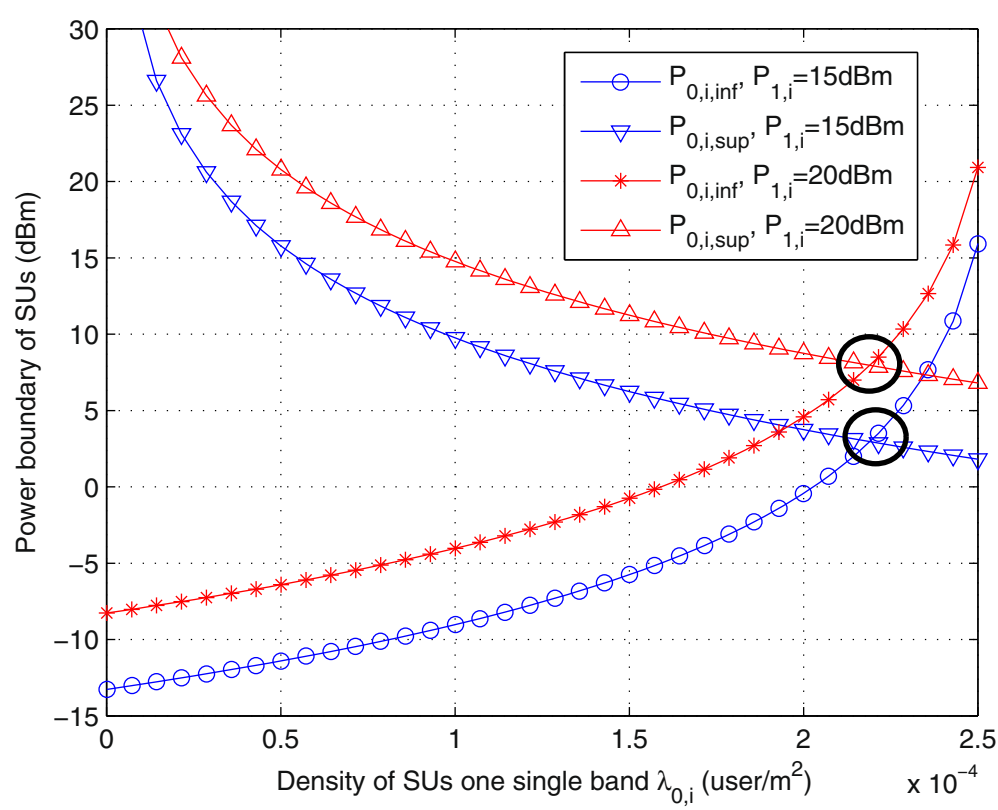

Fig. 5 Power boundary of SUs vs. density of SUs on one single band 


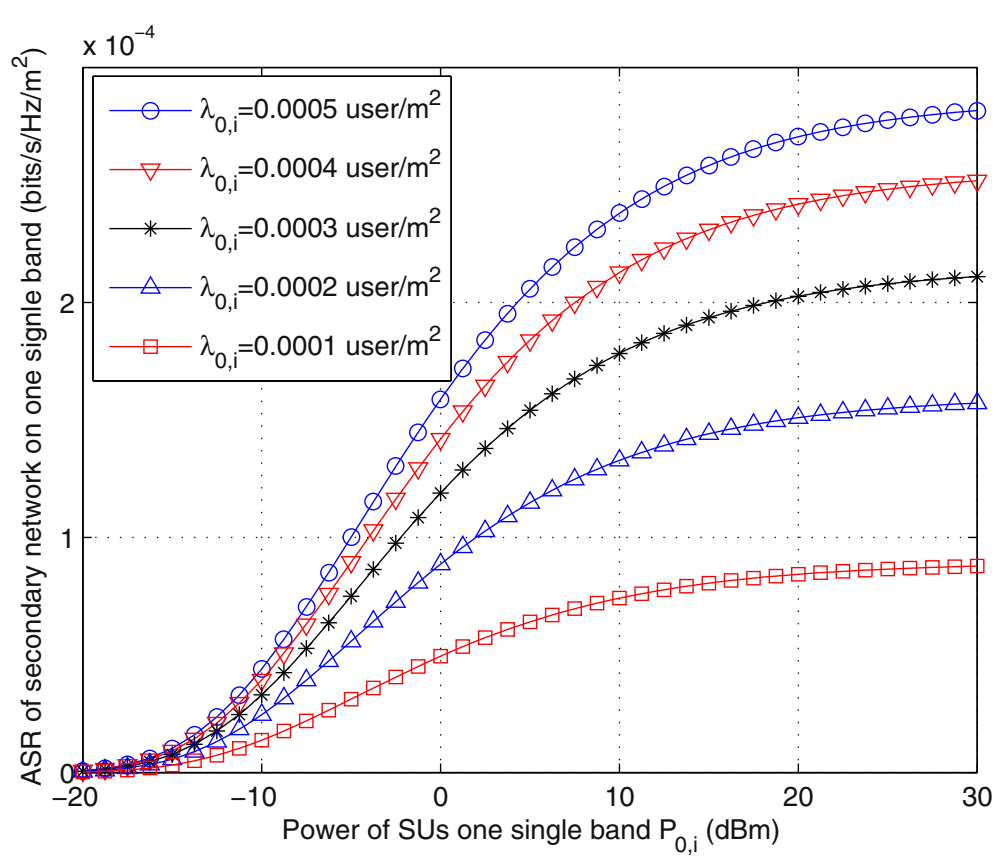

Fig. 6 ASR of the secondary network vs. power of SUs on one single band

Furthermore, the figure illustrates that when the power of PUs is decreasing, the ASR of SUs is rising, which is due to the reduction of interference from PUs to secondary network.

Next, the simulation results of CRNs on multiple bands are discussed. We also consider a simulation scenario as a $500 \times 500 \mathrm{~m}^{2}$ square region under the Monte Carlo simulation. The spectrum band of PUs is divided into five sub-bands with the bandwidth in three cases. Each band is assumed with a bandwidth normalized to 1 . The key parameters are listed in Table 2 . In cases 1 and 2, the average number of PUs on each band is 20 , while the average number of PUs on each band is 70 in case 3.

In Fig. 8, a comparison between the proposed algorithm and the average power allocation algorithm is illustrated.

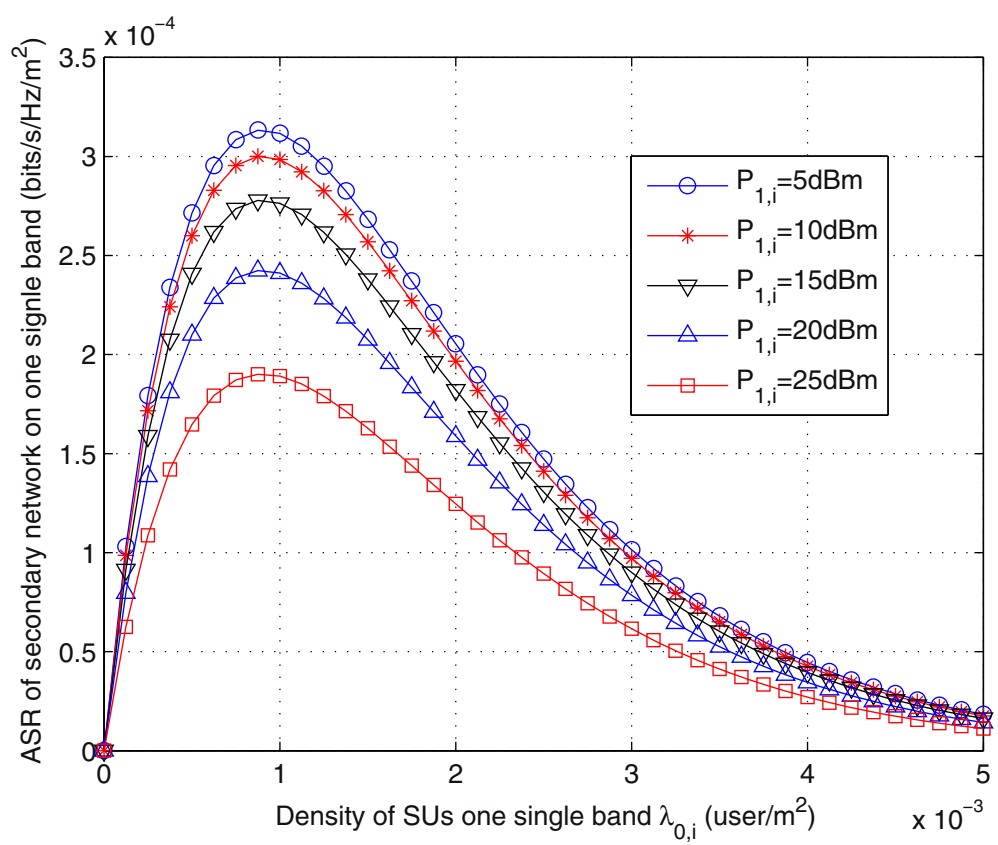

Fig. 7 ASR of secondary network vs. Density of SUs on one single band 


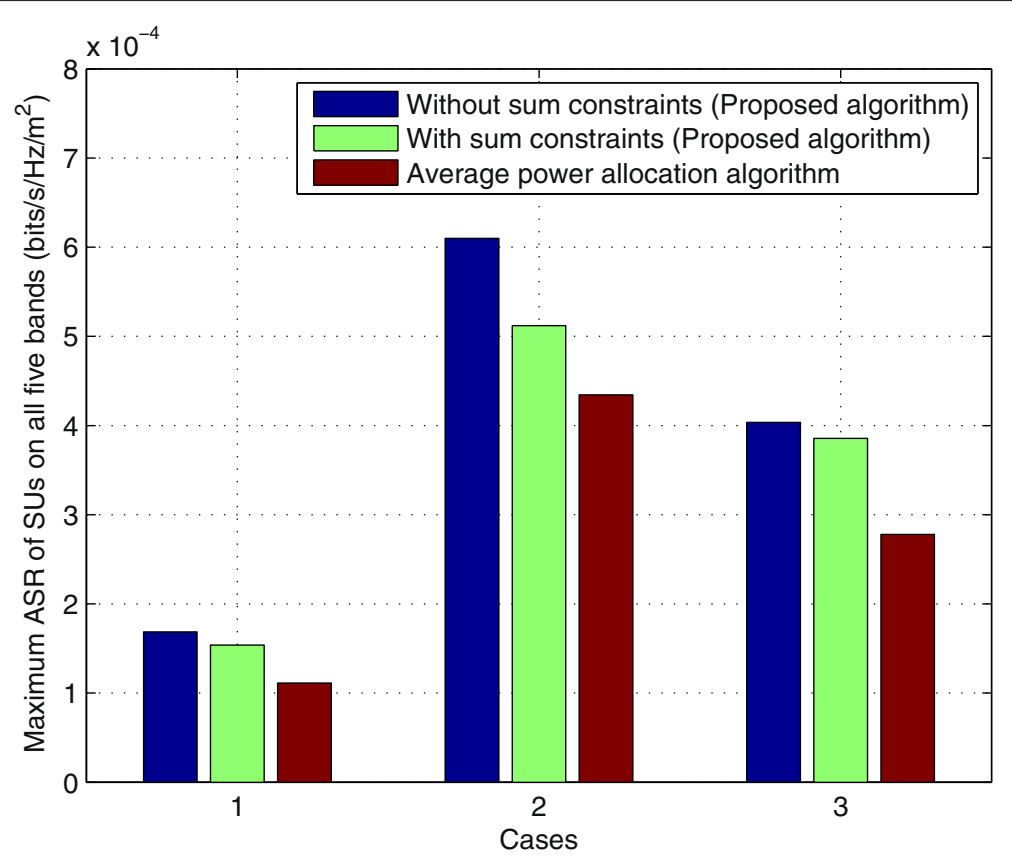

Fig. 8 Maximum ASR of secondary network on all five bands

The simulation is based on five bands with different densities and powers of PUs; the detailed parameters are shown in Table 2. Both algorithms adopt the same way to calculate the optimal density or transmission power of SUs. The only difference is when we calculate the density or transmission power of SUs in each iteration, the change in the quantity of density or power is distributed averagely to all the five bands with average power allocation algorithm. From Fig. 8, we can see that the sum of the ASR of the secondary network can achieve the highest values. While

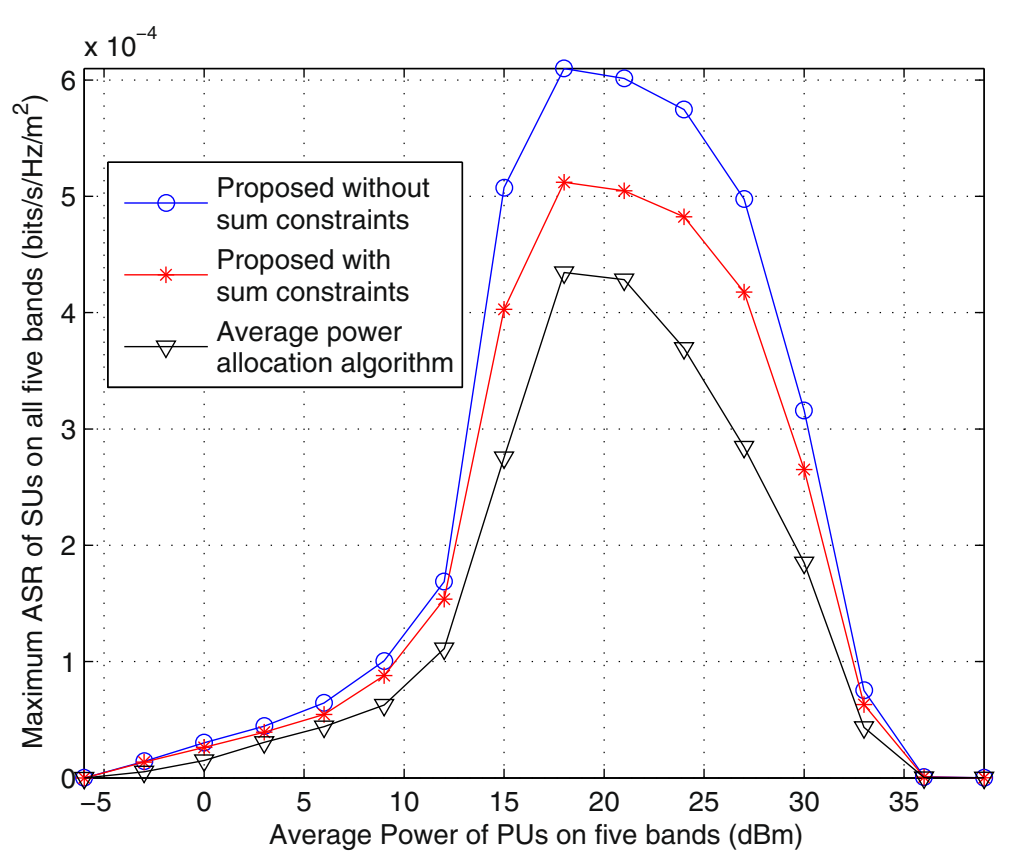

Fig. 9 Maximum ASR of SUs vs. average power of PUs on all five bands 
Table 2 Key parameters of the simulation on five bands

\begin{tabular}{|c|c|c|c|}
\hline \multirow[b]{2}{*}{ Parameter } & \multicolumn{3}{|c|}{ Parameters as [band 1 band 2 band 3 band 4 band5] } \\
\hline & Case 1 & Case 2 & Case 3 \\
\hline $\begin{array}{l}\text { Power of PUs } \\
(\mathrm{dBm})\end{array}$ & {$\left[\begin{array}{lllll}10 & 10 & 10 & 20 & 20\end{array}\right]$} & {$\left[\begin{array}{lllll}20 & 25 & 15 & 10 & 20\end{array}\right]$} & [20 251510 20] \\
\hline $\begin{array}{l}\text { Density of PUs } \\
\left(10^{-5} \cdot \text { user } / \mathrm{m}^{2}\right)\end{array}$ & {$\left[\begin{array}{lllll}1 & 1 & 1 & 1 & 3\end{array}\right]$} & {$\left[\begin{array}{lllll}1 & 1 & 1 & 1 & 3\end{array}\right]$} & [10 30502030$]$ \\
\hline
\end{tabular}

with the constraints of the sum of the density and power of SUs, the ASR is decreased due to the constraints from PUs that become strict. This forbids some SUs to access the spectrum of the PUs. Furthermore, we can see the proposed algorithm results to a better ASR of the secondary network, which is due to our proposed algorithm that can make a proper number of SUs to access the spectrum. In addition, compare case 1 with case 2 , we can see that PUs can endure more interference when the power of PUs becomes larger. So, more SUs can access the spectrum of PUs. But when both the density and power of PUs become large, the spectrum access of SUs is confined because the constraints of PUs become strict. Then, we can see that the ASR of the secondary network in case 3 is lower than that in case 2 .

Figure 9 shows the relationship between the maximum ASR of SUs and the average power of PUs on five bands. The densities of PUs on the five bands are as cases 1 and 2 have shown in Table 2. At first, we can see the maximum ASR of SUs is increasing as the average power of PUs is increasing. This is due to the fact that PUs cannot endure too much interference from SUs, the constraints to the SUs is strict, and the maximum ASR of SUs is also not very high. When the power continues to increase, the maximum ASR of SUs can reach its peak value. We can see our proposed algorithm is better than the average power allocation algorithm. In our proposed algorithm, a proper number of SUs access the spectrum of PUs. In addition, the maximum ASR is decreasing when the average power of PUs continues to increase. The interference from the PUs becomes serious when the average power of PUs becomes larger.

In Fig. 10, the maximum ASR of SUs is decreasing as the average density of PUs is decreasing. The power of PUs on the five bands are as cases 2 and 3 have shown in Table 2. As the black ellipse has shown, when the average density of PUs is low, the interference among the PUs is not high. Which means that when the SIR of PUs is high, PUs in the networks can endure more interference from the SUs. Thus, more SUs can access the spectrum of PUs, and the maximum ASR of SUs is high. But as the the density of PUs increases, the interference of PUs becomes large, which causes the constraints to SUs become strict. Then, we can see the curves decline. In addition, the proposed algorithm achieves a better performance than the average power allocation algorithm, which also verifies the results in Fig. 8 before.

\section{Conclusions}

In this paper, we have studied the optimal spectrum access and power control of SUs on multiple bands. The outage probabilities and ASR of SUs have been obtained based

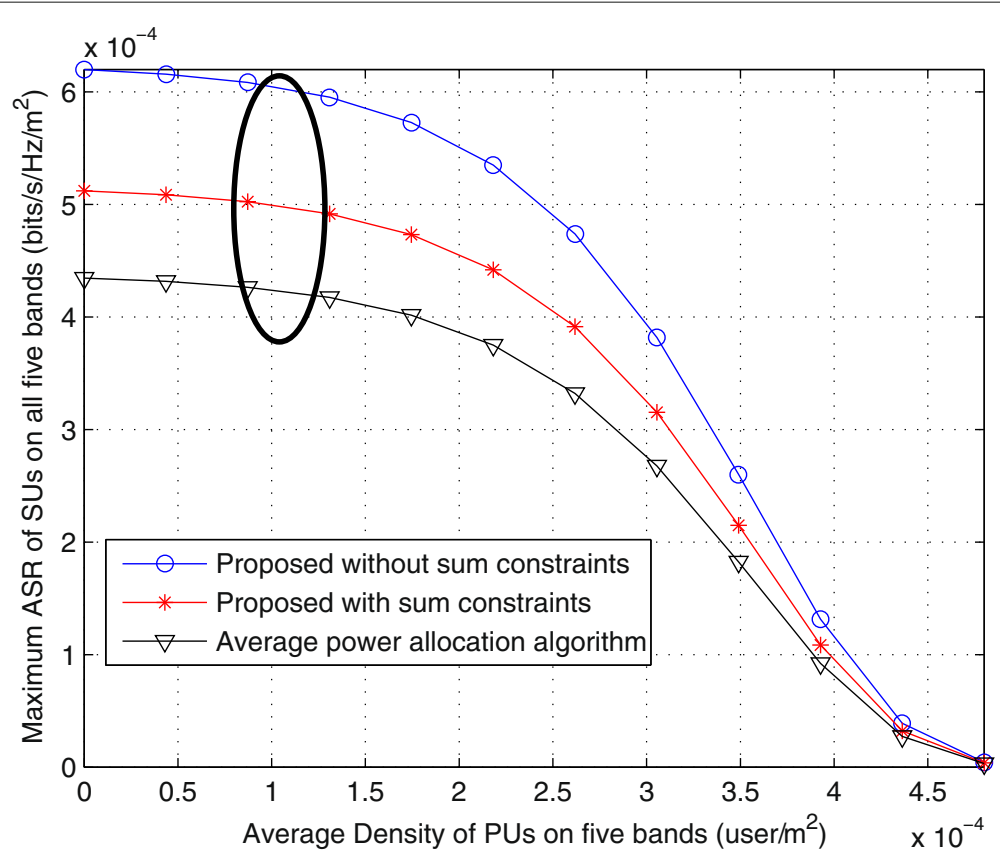

Fig. 10 Maximum ASR of SUs vs. average density of PUs on all five bands 
on the CRNs which are modeled by PPPs. Then, we have obtained the constraints from both PUs and SUs. The convexity of the target ASR has also been verified. So, we have derived out the optimal densities and powers of SUs in closed-form. Last, a spectrum access and power control algorithm of SUs has been proposed to get the maximum ASR of SUs. From the simulation, we can see that the outage probability, ASR, density, and power of SUs on each band are all constrained by the primary network and the interference in CRNs. The optimal values on multiple bands are reduced when the sum constraints are added. Simulation results have also verified the superiority of the proposed algorithm over the average power allocation algorithm.

\section{Appendix 1}

\section{Proof of Theorem 1}

Make $\lambda_{0, i}=x_{i}$, change the optimization problem (20) into the standard form as

$$
\begin{array}{cc}
\min & -f\left(x_{i}\right)= \\
\text { s.t. } & -\sum_{i=1}^{N} A_{i} x_{i} e^{-\varsigma_{0, i} x_{i}} \\
& x_{i} \geq 0 \\
& x_{i}-\lambda_{0, i, \mathrm{sup}} \leq 0,(i=1,2, \ldots, N) \\
& \sum_{i=1}^{N} x_{i}=\lambda_{0}
\end{array} .
$$

The second derivative of target function with respect to $\lambda_{0, i}$ satisfies

$$
-f^{\prime \prime}\left(x_{i}\right)=A_{i} \varsigma_{0, i}\left(2-\varsigma_{0, i} x_{i}\right) e^{-\varsigma_{0, i} x_{i}} .
$$

In practical CRNs, the outage probability thresholds $\theta_{0}$ and $\theta_{1}$ are both very small values, which leads to $\varsigma_{0, i} x_{i}<2$, so

$$
-f^{\prime \prime}\left(x_{i}\right)>0,0 \leq x_{i} \leq \lambda_{0, i, \text { sup }} .
$$

So, the maximization problem is a convex problem; define the symbol of optimal $x_{i}$ as $x_{i}^{*}$, then we have the Lagrange function as follows:

$$
\begin{aligned}
& L\left(x_{i}^{*}, k_{i}, l_{i}, v\right) \\
& =-\sum_{i=1}^{N} A_{i} x_{i}^{*} e^{-\varsigma_{0, i} x_{i}^{*}}-\sum_{i=1}^{N} k_{i} x_{i}^{*} \\
& \quad+\sum_{i=1}^{N} l_{i}\left(x_{i}^{*}-\lambda_{0, i, \mathrm{sup}}\right)+v\left(\sum_{i=1}^{N} x_{i}^{*}-\lambda_{0}\right) .
\end{aligned}
$$

According to the KKT condition, we get the following algebras:

(1) $x_{i}^{*} \geq 0, i=1,2, \ldots, N$;

(2) $k_{i} \geq 0, i=1,2, \ldots, N$;

(3) $l_{i} \geq 0, i=1,2, \ldots, N$;

(4) $x_{i}^{*}-\lambda_{0, i, \text { sup }} \leq 0, i=1,2, \ldots, N$;
(5) $k_{i} x_{i}^{*}=0, i=1,2, \ldots, N$;

(6) $l_{i}\left(x_{i}^{*}-\lambda_{0, i, \text { sup }}\right)=0, i=1,2, \ldots, N$;

(7) $-A_{i}\left(1-\varsigma_{0, i} x_{i}^{*}\right) e^{-\varsigma_{0, i} x_{i}^{*}}-k_{i}+l_{i}+v=0, i=$ $1,2, \ldots, N$

(8) $\sum_{i=1}^{N} x_{i}^{*}=\lambda_{0}$.

From (7) we have

$$
k_{i}=l_{i}+v-A_{i}\left(1-\varsigma_{0, i} x_{i}^{*}\right) e^{-\varsigma_{0, i} x_{i}^{*}} .
$$

From (6) we have

$$
l_{i} x_{i}^{*}=l_{i} \lambda_{0, i, \text { sup }}
$$

Take the two equations above into (5) and transform

$$
\left[v-A_{i}\left(1-\varsigma_{0, i} x_{i}^{*}\right) e^{-\varsigma_{0, i} x_{i}^{*}}\right] x_{i}^{*}+l_{i} \lambda_{0, i, \text { sup }}=0 .
$$

Combine with (1) to (5), we can know:

If $v \geq A_{i}, v-A_{i}\left(1-\varsigma_{0, i} x_{i}^{*}\right) e^{-\varsigma_{0, i} x_{i}^{*}}>0$, so $x_{i}^{*}=0, l_{i}=$ 0 .

If $v<A_{i}$, the following results are obtained:

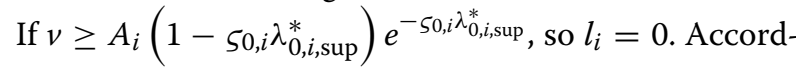
ing to (36), we have

$$
v-A_{i}\left(1-\varsigma_{0, i} x_{i}^{*}\right) e^{-\varsigma_{0, i} x_{i}^{*}}=0 .
$$

Bring $e^{-\varsigma_{0, i} x_{i}^{*}} \sim\left(1+\varsigma_{0, i} x_{i}^{*}\right)$ into the equation, we get $x_{i}^{*}=\frac{1}{\varsigma_{0, i}}\left(1-\sqrt{\frac{v}{A_{i}}}\right)$. Otherwise, we have $x_{i}^{*}=\lambda_{0, i, \text { sup }}$.

So from above, the results in Eq. (21) are obtained.

\section{Appendix 2}

Proof of Lemma 2

For the target function in (28), we get

$$
\begin{aligned}
& -\omega_{i} \lambda_{0, i} e^{-\varsigma_{0, i}\left[\lambda_{0, i}+\left(\frac{P_{1, i}}{P_{0, i}}\right)^{\frac{2}{\alpha}} \lambda_{1, i}\right]} \\
& =-\omega_{i} \lambda_{0, i} e^{-\zeta_{0, i} \lambda_{0, i}} \cdot e^{-\zeta_{0, i}\left(\frac{P_{1, i}}{P_{0, i}}\right)^{\frac{2}{\alpha}} \lambda_{1, i}} .
\end{aligned}
$$

Then the equation above can be transformed into $-f\left(\lambda_{0, i}, P_{0, i}\right)=-B_{i} e^{-D_{i} P_{0, i}^{\frac{-2}{\alpha}}}$. Calculate its second partial derivative with respective to $P_{0, i}$. We get

$$
\begin{aligned}
& -f^{\prime \prime}\left(\lambda_{0, i}, P_{0, i}\right) \\
& =\frac{2 B_{i} D_{i} e^{-D_{i} P_{0, i}^{\frac{-2}{\alpha}} P_{0, i}^{\frac{-2(\alpha+2)}{\alpha}}}}{\alpha^{2}}\left[-2 D_{i}+(\alpha+2) P_{0, i}^{\frac{2}{\alpha}}\right] .
\end{aligned}
$$

The domain of $P_{0, i}$ is $P_{0, i, \text { inf }} \leq P_{0, i} \leq P_{0, i, \text { sup }}$, and it is obvious that $-2 D_{i}+(\alpha+2) P_{0, i}^{\frac{2}{\alpha}}$ is monotonously increasing with $P_{0, i}$, so if the lower limit of $P_{0, i}$ makes the second partial derivative greater than zero, all the 
values of $P_{0, i}$ in the domain make so. Because $P_{0, i, \text { inf }}=$ $\left\{0, P_{1, i}\left[-\frac{\ln \left(1-\theta_{0}\right)}{\lambda_{1, i} S_{0, i}}-\frac{\lambda_{0, i}}{\lambda_{1, i}}\right]^{\frac{-\alpha}{2}}\right\}$, we have

(1) When $P_{0, i, \text { inf }}=0$, it is obvious that $-f^{\prime \prime}\left(\lambda_{0, i}, P_{0, i}\right) \geq$ 0 .

(2) When $P_{0, i, \text { inf }}>0$, we have

$$
\begin{aligned}
& -2 D_{i}+(\alpha+2) P_{0, i, \mathrm{inf}}^{\frac{2}{\alpha}} \\
& =-\varsigma_{0, i} P_{1, i}^{\frac{2}{\alpha}} \lambda_{1, i}\left[2+\frac{(\alpha+2)}{\ln \left(1-\theta_{0}\right)+\lambda_{0, i} \varsigma_{1, i}}\right] .
\end{aligned}
$$

From $P_{0, i, \text { inf }}>0$, we know

$$
P_{0, i, \text { inf }}=P_{1, i}\left[-\frac{\ln \left(1-\theta_{0}\right)}{\lambda_{1, i} \zeta_{0, i}}-\frac{\lambda_{0, i}}{\lambda_{1, i}}\right]^{\frac{-\alpha}{2}}>0 .
$$

So, we have

$$
P_{1, i}\left(\frac{1}{\lambda_{1, i}}\right)^{\frac{-\alpha}{2}}\left(\frac{1}{\varsigma_{0, i}}\right)^{\frac{-\alpha}{2}}\left[-\ln \left(1-\theta_{0}\right)-\varsigma_{0, i} \lambda_{0, i}\right]^{\frac{-\alpha}{2}}>0 .
$$

So, $\ln \left(1-\theta_{0}\right)+\varsigma_{0, i} \lambda_{0, i}<0$. The outage probability threshold of SUs $\theta_{0}$ is a very tiny value which ensures the communication in the secondary network. Here, we can make $0<\theta_{0}<1-e^{-\left(1+50, i \lambda_{0, i}\right)}$, so we get

$$
-1<\ln \left(1-\theta_{0}\right)+\varsigma_{0, i} \lambda_{0, i}<0 .
$$

Then, $2+\frac{\alpha+2}{\ln \left(1-\theta_{0}\right)+\zeta_{0, i} \lambda_{0, i}}<0$, and we have

$$
-2 D_{i}+(\alpha+2) P_{0, i, \mathrm{inf}}^{\frac{2}{\alpha}}>0 .
$$

Thus, we know $-f^{\prime \prime}\left(\lambda_{0, i}, P_{0, i}\right)>0$ when $P_{0, i}=$

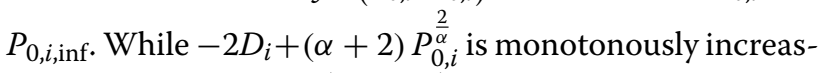
ing with $P_{0, i}$, so $-f\left(\lambda_{0, i}, P_{0, i}\right)$ is convex when $P_{0, i} \in$ $\left[P_{0, i, \text { inf }}, P_{0, i, \text { sup }}\right]$.

\section{Appendix 3}

\section{Proof of Theorem 2}

Make $P_{0, i}=p_{i}$, change the optimization problem (28) into the standard form as follows:

$$
\begin{gathered}
\min \quad-f\left(p_{i}\right)=-\sum_{i=1}^{N} \omega_{i} \lambda_{0, i} e^{-\varsigma 0, i}\left[\lambda_{0, i}+\left(\frac{P_{1, i}}{P_{0, i}}\right)^{\frac{2}{\alpha}} \lambda_{1, i}\right] \\
\text { s.t. } \quad p_{i}-P_{0, i, \text { inf }} \geq 0, i=1,2, \ldots, N \\
p_{i}-P_{0, i, \text { sup }} \leq 0, i=1,2, \ldots, N \\
\sum_{i=1}^{N} p_{i}=P_{0}
\end{gathered}
$$

From Lemma 2, we know the target function is convex. Define the symbol of optimal $p_{i}$ as $p_{i}^{*}$ and construct
Lagrange function as follows:

$$
\begin{aligned}
L\left(p_{i}^{*}, s_{i}, t_{i}, u\right) & \\
= & -\sum_{i=1}^{N} B_{i} e^{-D_{i} p_{i}^{*} \frac{-2}{\alpha}}-\sum_{i=1}^{N} s_{i}\left(p_{i}^{*}-P_{0, i, \mathrm{inf}}\right) \\
& +\sum_{i=1}^{N} t_{i}\left(p_{i}^{*}-P_{0, i, \mathrm{sup}}\right)+u\left(\sum_{i=1}^{N} p_{i}^{*}-P_{0}\right) .
\end{aligned}
$$

From KKT condition, we get

(1) $s_{i} \geq 0, i=1,2, \ldots, N$

(2) $t_{i} \geq 0, i=1,2, \ldots, N$

(3) $p_{i}^{*}-P_{0, i, \text { inf }} \geq 0, i=1,2, \ldots, N$

(4) $p_{i}^{*}-P_{0, i, \text { sup }} \leq 0, i=1,2, \ldots, N$

(5) $s_{i}\left(p_{i}^{*}-P_{0, i, \text { inf }}\right)=0, i=1,2, \ldots, N$

(6) $t_{i}\left(p_{i}^{*}-P_{0, i, \text { sup }}\right)=0, i=1,2, \ldots, N$

(7) $-\frac{2 B_{i} D_{i}}{\alpha} e^{-D_{i} p_{i}^{*} \frac{-2}{\alpha}} p_{i}^{*-\left(1+\frac{2}{\alpha}\right)}-s_{i}+t_{i}+u=0$;

(8) $\sum_{i=1}^{N} p_{i}^{*}=P_{0}$.

Transform (7) into

$$
s_{i}=-\frac{2 B_{i} D_{i}}{\alpha} e^{-D_{i} p_{i}^{*} \frac{-2}{\alpha}} p_{i}^{*-\left(1+\frac{2}{\alpha}\right)}+t_{i}+u .
$$

From (6), we have

$$
t_{i} p_{i}^{*}=t_{i} P_{0, i, \text { sup }}
$$

Substituting the two equations above into (5), we get

$$
\begin{aligned}
& {\left[u-\frac{2 B_{i} D_{i}}{\alpha} e^{-D_{i} p_{i}^{*} \frac{-2}{\alpha}} p_{i}^{*-\left(1+\frac{2}{\alpha}\right)}\right]\left(p_{i}^{*}-P_{0, i, \mathrm{inf}}\right)} \\
& \quad+t_{i}\left(P_{0, i, \text { sup }}-P_{0, i, \mathrm{inf}}\right)=0 .
\end{aligned}
$$

If $P_{0, i, \text { sup }} \leq P_{0, i, \text { inf }}$, we get $p_{i}^{*}=0$; otherwise, make $h\left(p_{i}^{*}\right)=\frac{2 B_{i} D_{i}}{\alpha} e^{-D_{i} p_{i}^{*} \frac{-2}{\alpha}} p_{i}^{*-\left(1+\frac{2}{\alpha}\right)}$, then it is a continuous function with its definition domain a compact closed set. So, defining its range $\left[h_{0, i, \min }, h_{0, i, \max }\right]$, we have the following:

(1) When $u \leq h_{0, i, \min }$, we have $p_{i}^{*}=P_{0, i, \text { inf }}, t_{i}=0$.

(2) When $h_{0, i, \min }<u \leq h_{0, i, \max }, t_{i}=0$, and $u-$ $\frac{2 B_{i} D_{i}}{\alpha} e^{-D_{i} p_{i}^{*} \frac{-2}{\alpha}} p_{i}^{*-\left(1+\frac{2}{\alpha}\right)}=0$. According to equivalent infinite $e^{-D_{i} p_{i}^{*} \frac{-2}{\alpha}} \sim\left(1-D_{i} p_{i}^{*} \frac{-2}{\alpha}\right)$ and substitute it into the equation before, we have $u-\frac{2 B_{i} D_{i}}{\alpha}(1-$ $\left.D_{i} p_{i}^{* \frac{-2}{\alpha}}\right) p_{i}^{*-\left(1+\frac{2}{\alpha}\right)}=0$. When all the parameters are fixed, the numerical solution of $p_{i}^{*}$ can be obtained, and we define it as $P_{0, i, \text { solution }}^{*}$

(3) When $u>h_{0, i, \text { max }}$, we have $p_{i}^{*}=P_{0, i, \text { sup }}$ which satisfies $u-\frac{2 B_{i} D_{i}}{\alpha} e^{-D_{i} P_{0, i, s u p}^{-\frac{2}{\alpha}}} P_{0, i, \text { sup }}^{-\left(1+\frac{2}{\alpha}\right)}+t_{i}=0$. 
Substitute the results above into (8), i.e., $\sum_{i=1}^{N} p_{i}^{*}=P_{0}$. We can get the numerical solution of $u$, so we can obtain the specific value of $p_{i}^{*}$ on each band.

\section{Acknowledgements}

This work was supported by the the National Key Basic Research Program of China (Grant No. 2013CB329203), the National Natural Science Foundation of China (Grant Nos. 61571270 and 61271266), the China Postdoctoral Science Foundation (Grant No. 2016M591177), and the British Telecom and Tsinghua SEM Advanced ICT LAB. The research leading to these results also received funding from the European Commission H2020 programme under grant agreement no. 671705 (SPEED-5G project).

\section{Competing interests}

The authors declare that they have no competing interests.

\section{Publisher's Note}

Springer Nature remains neutral with regard to jurisdictional claims in published maps and institutional affiliations.

\section{Author details}

${ }^{1}$ Tsinghua National Laboratory for Information Science and Technology (TNList), Department of Electronic Engineering, Tsinghua University, 100084 Beijing, China. ${ }^{2}$ School of Electric and Information Engineering, Zhongyuan University of Technology, 450007 Zhengzhou, China. ${ }^{3}$ Instituto de Telecomunicações, 1049-001 Aveiro, Portugal.

\section{Received: 30 October 2016 Accepted: 5 May 2017}

Published online: 30 May 2017

\section{References}

1. A Gupta, RK Jha, A survey of $5 \mathrm{G}$ network: architecture and emerging technologies. IEEE Access. 3, 1206-1232 (2015)

2. S Haykin, Cognitive radio: brain-empowered wireless communications. IEEE J. on Sel. Areas Commun. 23(2), 201-220 (2005)

3. F-H Tseng, H-c Chao, J Wang, et al., Ultra-dense small cell planning using cognitive radio network toward 5G. IEEE Wirel. Commun. 22(6), 76-83 (2015)

4. C-h Lee, M Haenggi, Interference and outage in poisson cognitive networks. IEEE Trans. Wirel. Commun. 11(4), 1392-1401 (2012)

5. P Madhusudhanan, L Youjian, TX Brown, On primary user coverage probabilities and faulty cognitive radios. IEEE Trans. Wirel. Commun. 13(11), 6207-6218 (2014)

6. $X X u, W L i u, Y$ Cai, S Jin, On the secure spectral-energy efficiency tradeoff in random cognitive radio networks. IEEE J. on Sel. Areas Commun. 34(10), 2706-2722 (2016)

7. H Shokri-Ghadikolaei, I Glaropoulos, V Fodor, C Fischione, A Ephremides, Green sensing and access: energy-throughput trade-offs in cognitive networking. IEEE Commun. Mag. 53(11), 199-207 (2015)

8. X Hong, J Wang, C-X Wang, J Shi, Cognitive radio in 5G: a perspective on energy-spectral efficiency trade-off. IEEE Commun. Mag. 52(7), 46-53 (2014)

9. M Yousefvand, N Ansari, S Khorsandi, Maximizing network capacity of cognitive radio networks by capacity-aware spectrum allocation. IEEE Trans. Wirel. Commun. 14(9), 5058-5067 (2015)

10. J Mansukhani, P Ray, PK Varshney, Coupled detection and estimation based censored spectrum sharing in cognitive radio networks. IEEE Trans. Wirel. Commun. 15(6), 4206-4217 (2016)

11. W Wang, L Wu, Z Zhang, L Chen, Joint spectrum sensing and access for stable dynamic spectrum aggregation. EURASIP J. Wirel. Commun. Netw. 2015(130), 1-14 (2015)

12. X Zhang, H Su, Opportunistic spectrum sharing schemes for CDMA-based uplink mac in cognitive radio networks. IEEE J. on Sel. Areas Commun. 29(4), 716-730 (2011)

13. J Huang, VG Subramanian, R Agrawal, RA Berry, Downlink scheduling and resource allocation for OFDM systems. IEEE Trans. Wirel. Commun. 8(1), 288-296 (2009)
14. Z Dai, Z Wang, V Wong, An overlapping coalitional game for cooperative spectrum sensing and access in cognitive radio networks. IEEE Trans. Veh. Technol. 65(10), 8400-8413 (2016)

15. X Yuan, F Tian, YT Hou, W Lou, HD Sherali, S Kompella, JH Reed, in Proceedings of IEEE International Symposium on Dynamic Spectrum Access Networks (DySPAN). Optimal throughput curve for primary and secondary users with node-level cooperation, (Stockholm, 2015), pp. 358-364

16. D-J Lee, Adaptive random access for cooperative spectrum sensing in cognitive radio networks. IEEE Trans. Wirel. Commun. 14(2), 831-840 (2015)

17. AV Kordali, PG Cottis, A contract-based spectrum trading scheme for cognitive radio networks enabling hybrid access. IEEE Access. $\mathbf{3}$ $1531-1540$ (2015)

18. G Hattab, M Ibnkahla, Multiband spectrum access: great promises for future cognitive radio networks. Proc. IEEE. 102(3), 282-306 (2014)

19. TMC Chu, H Phan, H-J Zepernick, Hybrid interweave-underlay spectrum access for cognitive cooperative radio networks. IEEE Trans. Wirel. Commun. 62(7), 2183-2197 (2014)

20. CWang, S Tang, X-Y Li, C Jiang, Multicast capacity scaling laws for multihop cognitive networks. IEEE Trans. Mob. Comput. 11(11), 1627-1639 (2012)

21. N Rahimian, C N. Georghiades, M Z. Shakir, K A. Qaraqe, On the probabilistic model for primary and secondary user activity for OFDMA-based cognitive radio systems: spectrum occupancy and system throughput perspectives. IEEE Trans. Wirel. Commun. 13(1), 356-369 (2014)

22. S Kusaladharma, P Herath, C Tellambura, Underlay interference analysis of power control and receiver association schemes. IEEE Trans. Veh. Technol. 65(11), 8978-8991 (2016)

23. F Baccelli, B Błaszczyszyn, Stochastic Geometry and Wireless Networks Volumel: Theory. (NOW: Foundations and Trends in Networking, Pairs, 2009)

24. M Haenggi, Stochastic Geometry for Wireless Networks. (Cambridge University Press, New York, 2012)

25. Q Ye, M Al-Shalash, C Caramanis, JG Andrews, in Proceedings of IEEE International Conference on Communications (ICC). A tractable model for optimizing device-to-device communications in downlink cellular networks, (Sydney, 2014), pp. 2039-2044

\section{Submit your manuscript to a SpringerOpen ${ }^{\circ}$ journal and benefit from:}

- Convenient online submission

- Rigorous peer review

- Immediate publication on acceptance

- Open access: articles freely available online

- High visibility within the field

- Retaining the copyright to your article

Submit your next manuscript at $\mathbf{s p r i n g e r o p e n . c o m ~}$ 